\title{
A META-MODEL FOR THE DIALOG FLOW NOTATION
}

\author{
Matthias Book, Volker Gruhn \\ Chair of Applied Telematics / e-Business, University of Leipzig \\ Klostergasse 3, 04109 Leipzig, Germany \\ Email: \{book, gruhn\}@ebus.informatik.uni-leipzig.de \\ Nils Mirbach \\ adesso $A G$ \\ Stockholmer Allee 24, 44269 Dortmund, Germany \\ Email: mirbach@adesso.de
}

\begin{abstract}
Keywords: Web engineering, Dialog Flow Notation, metamodelling.
Abstract: While the separation of presentation and application logic is widely practiced in web-based applications today, many do not cleanly separate application and dialog control logic, which leads to inflexible implementations especially when multiple presentation channels shall be served by the same application logic. We therefore present a notation for specifying the complete dialog flow of an application separately from the application logic and show how to construct a formal metamodel for it using the OMG's Meta-Object Facility (MOF). This allows the validation of dialog flow models, as well as the generation of machine-readable dialog flow specifications from graphical models.
\end{abstract}

\section{INTRODUCTION}

Complex web-based applications are typically implemented according to the Front Controller pattern (Singh et al., 2002) today. While it follows the ModelView-Controller (MVC) paradigm (Krasner, 1988), many applications do not cleanly separate application, presentation and control logic when using this approach. In the popular Struts Web Application Framework (Apache Project, 2005), for example, the controller dispatches incoming requests to actions that implement or invoke the application logic. Afterwards, they tell the controller which view to display next, and the controller forwards the request to the appropriate page. Since the next page to display is determined by individual actions, the dialog control logic is distributed over all actions and entwined with the application logic inside them.

This combination leads to a number of problems: Firstly, the actions can only make relatively isolated and local dialog flow decisions, but are unaware of the "big picture" of the application's complete dialog flow. Secondly, the hard-coded decentralized implementation of the dialog control logic is relatively inflexible, almost unsuitable for reuse and hard to maintain or extend. Finally, achieving device independence would require additional effort and possibly redundant work: While the dialog flows for various client devices may differ depending on their in- put/output capabilities, the application logic should be independent of those specifics - however, their close coupling may prevent the reuse of actions on multiple presentation channels. Instead, each presentation channel would require its own set of actions to implement the individual dialog flows for the respective devices.

To address these challenges, we already presented a Dialog Control Framework (DCF) (Book and Gruhn, 2003) that decouples not only application and presentation logic, but also dialog control logic and dialog flow specification from each other. In this approach, pages (or dialog masks, as we call them) and actions do not explicitly call other masks and actions, but generate events that are handled by a central dialog controller. The dialog controller determines the receivers of events by looking them up in an object-oriented model of the whole application's dialog flow, and then calls the mask or action specified in the model. This awareness of the "big picture" enables the dialog controller to manage complex dialog structures - in particular, it allows the nesting of so-called dialog modules at arbitrary levels. The strict separation of dialog control logic and application logic also enables convenient reuse of actions in different dialog flows adapted for various devices.

Having such a framework that can be reused as a black box to control the dialog flow of any web-based application, we just need a way of providing it with a 
model of that dialog flow in an efficient manner. For this purpose, we developed the Dialog Flow Notation (DFN), a graphical notation that allows the specification of complex, modular dialog flows for multiple presentation channels (Book and Gruhn, 2004). The DFN was developed with the aim of producing implementable specifications: Its diagrams should not merely have descriptive, but also constructive power and be directly translatable into a dialog flow model that can be used by the framework. This way, we strive to reduce the development effort and eliminate the error sources that a manual re-implementation of the modeled dialog flows would incur.

The Object Modeling Group's model-driven development (MDD) approach seems well-suited to achieve this goal: Its Meta-Object Facility (MOF) (Object Management Group, 2002) enables us to create a Dialog Flow Metamodel that formally defines all elements of the DFN and the constraints that need to be satisfied when building dialog graphs with it. Using this metamodel, we can equip a graphical modeling tool with semantic awareness about the dialog flow models that users design, enabling it to check the models for syntactic and semantic validity and to generate machine-readable specifications from the model.

In this paper, after an overview of related work on the subject of web application modeling (section 2), we will give a brief introduction into the Dialog Flow Notation (section 3) and then present its meta model (section 4) along with a discussion of the issues that had to be resolved in its design. Using the DFN meta model, we implemented a plugin for the Eclipse IDE that allows the graphical modeling of dialog flows. We conclude with a brief presentation of this tool and an outlook on further opportunities for using the meta model in dialog flow specification and dialog control.

\section{RELATED WORK}

There is a number of ongoing efforts to model web applications using UML. (Conallen, 1999) defined a UML profile with stereotypes for components, classes, methods and association in order to distinguish server and client components, server pages etc. This approach comprises modeling both layout and implementation aspects, but does not support modularisation except by the use of frames.

Although they extend Conallen's UML profile, (Gorshkova and Novikov, 2002) do not add a means for modularisation. Instead, they focus on modeling the user interface and separate its functional model from its presentation characteristics. This way, they reach a more robust model, which is restricted to design-time use, however. Both approaches share the advantage of UML tool support for modeling, but also the disadvantage of lacking modularisation and constructive use of the models in application development.

In order to employ the models in the immediate implementation of the application, semantic information in the form of a meta-model is necessary. (Muller et al., 2003) examined how the MDA vision can be applied to web engineering and developed a platformindependent model for web applications that allows the generation of whole executable applications. The complete model of such an application consists of three parts: the business model, the hypertext model and the presentation model, which are similar to the actions, dialog flow and masks of the Dialog Flow Notation used in our approach.

The approach of Muller et al. goes a step further than e.g. (Schattkowsky and Lohmann, 2002), who developed a UML profile for small and mediumsized web applications. When developing web applications with their profile, code fragments for business logic and presentation logic can be generated from the model as a starting point for further implementation by the developers. After this big generation step, the models are discarded. In contrast, the dialog flow model introduced in this paper is updated together with the application logic and used continuously at run-time.

A big generation step is also characteristic of the Web Modeling Language (WebML) (Ceri et al., 2000), which supports the specification of complex web pages on a conceptual level. WebML distinguishes a structural model, a composition model and the topology of links between pages. In addition, it allows developers to define layout requirements and features a personalisation model. However, WebML is a proprietary language with its own XML representation and code generation tools, and therefore does not belong to the MDA standard.

\section{DIALOG FLOW NOTATION}

The Dialog Flow Notation (DFN) specifies the sequence of UI pages and processing steps in an application, and the data exchanged between them. It models the dialog flow as a transition network called a dialog graph. The notation refers to the transitions as events and to the states as dialog elements. These elements are further divided into hypertext pages (symbolized by dog-eared sheets and referred to by the more generic term masks in the DFN) and business logic operations (symbolized by circles and called actions here). Every dialog element can generate and receive multiple events. Which element will receive an event depends both on the event and the generating 


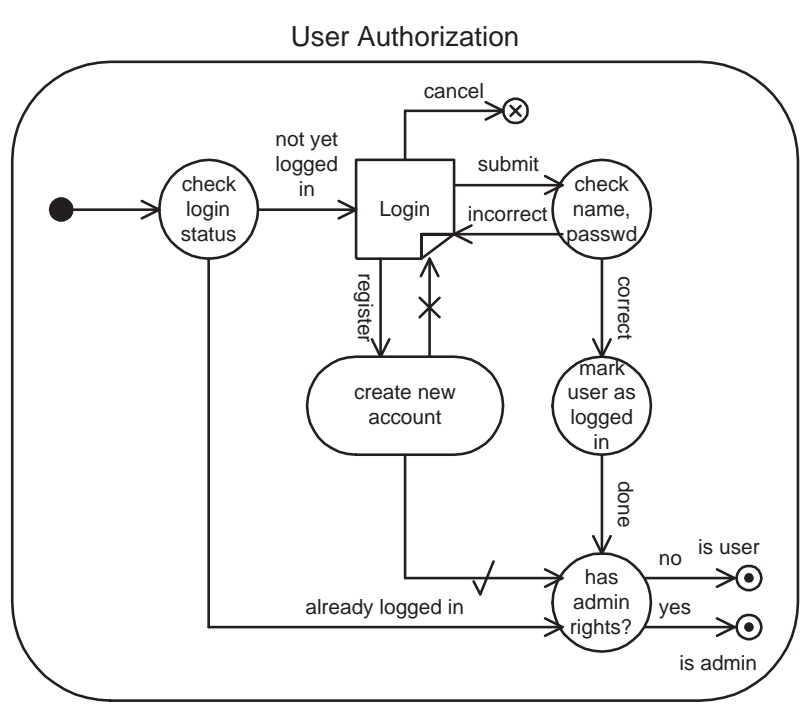

Figure 1: User Authorization dialog module

element (e.g., an event $e$ may be received by action $a_{1}$ if it was generated by mask $M_{1}$, but be received by action $a_{2}$ if generated by mask $M_{2}$ ). Events can carry parameters containing form input submitted through a mask or data produced by the business logic to facilitate communication between elements. They are not bound to HTTP requests or responses, but can also link two actions or two masks.

The DFN also provides dialog modules (symbolized by boxes with rounded corners) which encapsulate dialog graphs and enable the specification of nested dialog structures. When a module receives an event from the exterior dialog graph that it is embedded in, traversal of its interior dialog graph starts with the initial event. When the interior dialog graph terminates, it generates a terminal event (symbolized by a circular anchor point in the interior dialog graph and an arrow in the exterior dialog graph) that is propagated to the super-module and continues the traversal of the exterior dialog graph (Figure 1). For more complex dialog structures, the DFN offers a number of additional event and element types (Book and Gruhn, 2004) that we will not discuss here in detail for the sake of brevity.

To cater to the different interaction patters required for different client devices, the DFN allows the specification of dialog flows for different presentation channels in multiple versions of a module and distinguishing them with channel labels (Figure 2). While the channels employ different dialog masks according to those devices' I/O capabilities, they use the same actions for processing the users' input, as indicated by the shading. This enables developers to reuse the device-independent business logic on multiple chan-

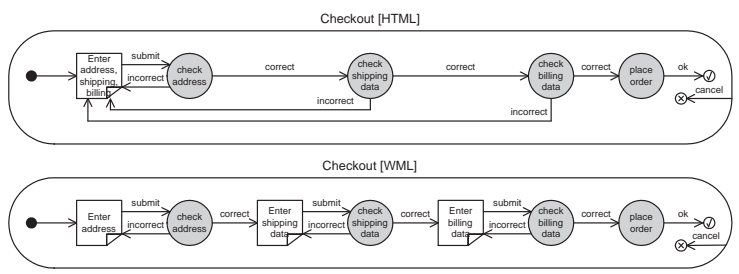

Figure 2: Checkout dialog module variants for HTML and WML presentation channel

nels.

In order to support an efficient transition from specification to implementation of dialog flows, we developed the Dialog Flow Metamodel described in the following section. It formally describes all elements and constraints of the DFN and enables us to generate machine-readable specifications in the XML-based Dialog Flow Specification Language (DFSL) from graphical dialog flow models. These specifications can then be parsed by the Dialog Control Framework (DCF) which serves as an application- and deviceindependent interface between presentation and business logic.

\section{DIALOG FLOW METAMODEL}

Without a formal specification of the DFN, the dialog flow models could not be used directly for dialog control since the graphical diagrams would have to be translated manually into the corresponding DFSL representation required by the DCF. This slow and errorprone process of dealing with two unvalidated and unsynchronized representations of the same model would reduce the efficiency of the software development process considerably. To enable automatic validation and transformation of the model representations, we developed the DFN metamodel. In this process, we had to resolve three issues that are discussed in the following sections:

\subsection{Formal Specification}

The first issue was to find a suitable formalism for the complete description of DFN models with their rich syntax and semantics. We chose the Meta-Object Facility (MOF) standard (Object Management Group, 2002) which defines formal metamodels for platformindependent metadata and mappings to specific platforms. MOF uses an object modelling framework for the definition of information models for metadata, which essentially is a subset of UML class diagrams that provides four main concepts for modelling: Classes model MOF meta-objects, associations model binary connections between them, data 
types model primitive or external data, and packages unitize the models. The Essential MOF (EMOF) standard and the Eclipse Modeling Framework (EMF), its implementation on the Eclipse platform, provide only a subset of these constructs that is sufficient for simple and pragmatic metamodelling. While EMOF proved to be mostly rich enough to describe all constructs of the DFN, workarounds had to be found for some aspects:

- Hierarchical structure: While the DFN supports and encourages the specification of dialog modules that are nested into each other at run-time, the module specifications themselves are not hierarchical in nature (in analogy to objects' methods that may also call each other at run-time without exhibiting a hierarchical relationship in their implementation). Since the metamodel requires its elements to be hierarchically structured in order to be displayed and serialized properly, however, any references to submodules inside a module were modeled as siblings of the actual module definitions at the root level.

- Graphical representation: Since the DFN itself does not contain any information about the graphical representation and layout of the individual notation elements in a diagram, this information had to be supplemented in the metamodel. Each notation element received additional position and size attributes in order to make this information persistent and displayable. This could be realized either by incorporating the layout information directly into the metamodel or by specifying the necessary information separately in its own package. To avoid the unnecessary complexity of cross-package references, we chose to specify the layout information directly in the metamodel.

- Clarity: Convenience and clarity also affected the design in other ways. The DFN provides various types of events which connect various types of model elements under certain constraints. An explicit specification of all these $n: n$ relations would have "polluted" the metamodel with many associations. To retain clarity, we implemented the constraints in the model elements themselves and used inheritance to propagate them among similar classes of elements: Every model element has an isTargetFor and an issourceFor method that decide if a certain event can be used to connect this element to a certain other element.

- Constraints: More important than the constraints that were implemented instead of being modeled for reasons of clarity are those constraints which are defined in the DFN itself. From widely used constraints for multiplicity, which can be designed into the metamodel itself, to complex, contextsensitive ones, constraints are the heart of valida- tion in metamodelling. We will discuss their implementation in more detail in the following section.

\subsection{Constraint Implementation}

With the DFN metamodel being expressive enough to cover every correct DFN model, the second challenge was the formalization of constraints that must be enforced on the model to ensure its validity.

Simple constraints are easy to specify within the metamodel by using MOF constructs like associations and multiplicities. These kinds of constraints are easily designed by connecting model elements, but they can decrease the clarity. To enforce more complex notation rules, constraints have to be implemented in the model elements themselves. As an example for a relatively simple constraint implementation, consider the following isTargetFor method of the Action class, specifying that actions can receive terminal, compound, initial and abort events, but no other event types:

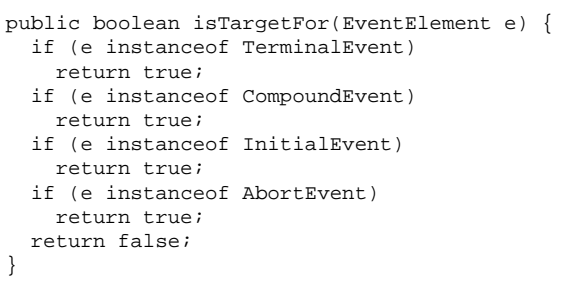

The following more complex example implements a constraint which determines that a Module element can only generate a terminal event if it is not a socalled common compound and if its dialog graph contains a suitable Terminal Anchor element. Since these conditions are context-sensitive (i.e. they depend on the presence and attributes of other elements that the designer may use in a concrete model), they cannot be expressed in terms of metamodel constructs but must be implemented in a validation routine of the respective metamodel element. The following excerpt from the issourceFor method of the Module class illustrates their implementation:

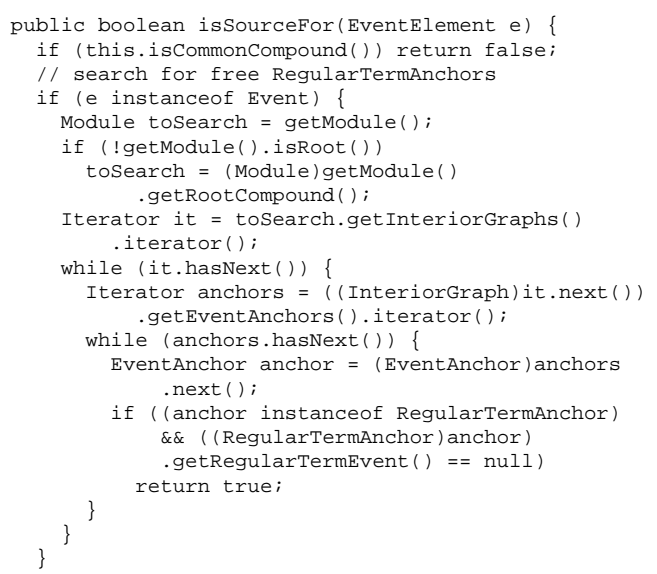


// $\ldots$

The EMF's Validation Framework uses both the simple constraint specifications expressed in the metamodel and the complex constraint implementations in the isTargetFor and isSourceFor methods to identify invalid constructs in DFN models and highlight them in the graphical editor.

\subsection{Model Transformation}

The third implementation challenge was the transformation of the valid DFN model into DFSL documents that can be interpreted by the Dialog Control Framework. Based on the structural information in the DFN metamodel, each concrete DFN model can automatically be serialized in the XML Metadata Interchange (XMI) 2.0 format by the EMF. The resulting file is human-readable, but does not require any manual processing to make DFN models machinereadable, which removes a slow and error-prone step from the application development process.

Since the DCF does not yet support the OMG standard XMI 2.0, the specification still has to be translated into the Dialog Flow Specification Language understood by the framework. For this purpose, we chose XSLT as the transformation language for several reasons: Firstly, there is a direct mapping from XMI to DFSL since the DFSL only uses a subset of the semantic data, so the transformation only involves reducing the XMI document's contents to the data required by the framework and building a new XML file from it using DFSL syntax. Possible synchronization problems between the two representations of a DFN model, caused by the batch-like execution of XSLT, do not occur due to the nature of the development process, which requires only one transformation before executing the dialog flow in the framework.

The final transformation is included in the development process of web applications through the integration of an export wizard into the DFN editor.

\subsection{Metamodel Elements}

After discussing the major issues encountered in the design of the metamodel, some worthwhile aspects regarding individual model elements should be mentioned here. Figure 3 shows an excerpt from the complete metamodel that contains classes modeling dia$\log$ elements.

The Element class plays a central role in the DFN metamodel. An Element has a position and a size which are used to display it in a graphical editor, as well as constraints for linkage with events defined in the isTargetFor and is SourceFor methods.

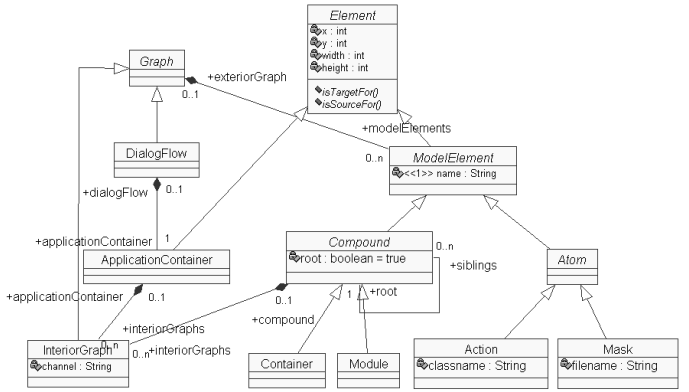

Figure 3: Metamodel excerpt: Dialog elements

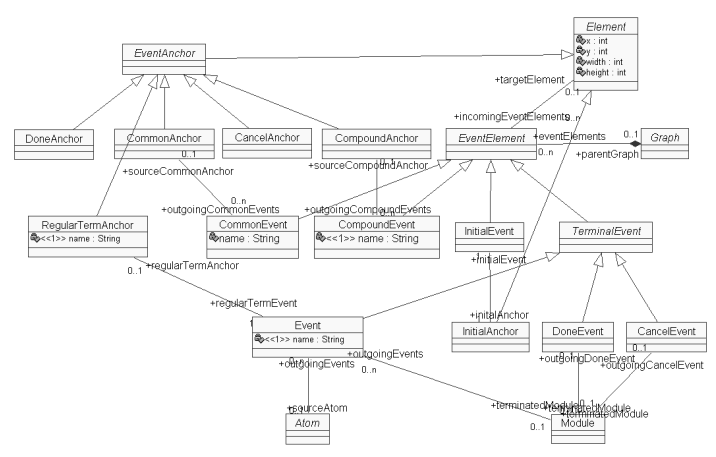

Figure 4: Metamodel excerpt: Dialog events

These characteristics are inherited by every Element subclass.

Most atomic and compound notation elements (masks, actions, modules and containers) are modeled as subclasses of the ModelElement class, where the Compound class has a self-reference to itself to realize the sibling relationship described in section 4.1. Also, the so-called application container that contains the dialog graph the user will initially traverse upon entering a web-based application is modeled separately here to put it on top of the metamodel's class hierarchy. Both compounds and the application container can contain multiple interior dialog graphs that in turn contain model elements. By giving different channel identifiers to different InteriorGraph instances, we can specify variants of the same dialog graph for different devices.

This containment hierarchy is a central concept of the DFN metamodel that is used both by the graphical representation and the serialization mechanism. Apart from these containment relations, model elements are also characterized by the type of their incoming and outgoing events. These are represented in the metamodel by the classes shown in Figure 4.

As mentioned before, inheritance was used to express the relations between elements and events for 
the sake of clarity. This is reflected in the metamodel by specifying the targetElement association only between the EventElement and Element classes, and letting all other event classes inherit this characteristic from EventElement. Since this construct potentially allows any element to receive any event, constraints for the allowed combinations must be implementated in the isTargetFor method, as shown in section 4.2.

Another worthwhile aspect to mention is the use of anchors within the metamodel. While e.g. terminal events use anchors in the interior graph to connect the dialog flow with the exterior graph, other events that are called directly by the DCF (such as the initial event) were originally drawn without an anchor. For a consistent representation in the metamodel, we added corresponding anchor elements to the metamodel for every event that is not generated or received by a regular element. This way, events in DFN models always connect two elements.

The graphical editor provides the advantages commonly associated with metamodelling: It supports modelling and enables automatic validation and transformation of domain-specific concepts, with the latter two steps being essential for increased efficiency in the software development process compared to a manual approach. If the DCF supported the DFN metamodel directly, it could have been integrated with the DFN editor, and the additional transformation step into the DFSL could also have been avoided.

\subsection{Metamodel vs. UML Profile}

As a meta-metamodel, MOF is capable of describing new metamodels, as shown in Fig. 5. However, domain-specific modeling could also be achieved by extending UML through its profile mechanism. Having described the DFN metamodel, one might therefore ask why we did not choose to specify the DFN using an UML profile instead of developing our own metamodel. To answer this question, we need to consider which way provides the most benefit for modellers and developers.

(Muller et al., 2003) propose that the development of a new metamodel is justified if the semantic distance between UML and the developed language is too large, and (Desfray, 2000) holds that the definition of a new metamodel is justified if the described domain is well-defined and possesses its own accepted quantity of main concepts.

Since the DFN was inspired by Statecharts (Harel, 1987 ) and their concepts resemble each other (apart from semantic peculiarities of the dialog control domain), a solution using UML profiles could have been chosen in this case. However, we chose to develop a new metamodel for several reasons: The DFN applies to the well-defined domain of web application development, which qualifies it for a metamodel of its own, according to Desfray. In addition, UML profiles have some disadvantages compared to new metamodels, e.g. insufficient transitivity in the derivation of properties (Atkinson et al., 2000).

In addition to these more academic reasons, there were other, more pragmatic ones: The biggest advantage of UML could be the support of many tools that are provided by different vendors. Unfortunately, though, there do not seem to be any robust tools that provide UML 2.0 profile support, which negates the biggest advantage of UML profiles. Pending a solution for this problem in the future, it would be interesting to examine whether the DFN metamodel can be represented as an UML profile and whether the modelling tools available then can significantly increase productivity. At this time, however, we found that the combination of Eclipse's EMF and Graphical Editing Framework (GEF) provides comprehensive support for the implementation of a CASE tool for the DFN based on a new metamodel.

\section{DFN CASE Tool}

To demonstrate the feasibility of our approach to dialog flow specification and evaluate the advantages and drawbacks of metamodelling, we implemented a CASE tool for the DFN. Our plugin for the Eclipse IDE can manipulate DFN models, validate them and transform them into DFSL documents.

The choice of Eclipse as the development platform was influenced by (Lüer, 2003), who judged it as the perfect candidate for the integration of experimental tools. The DFN CASE tool was developed as two Eclipse plugins - the DFN metamodel itself, implemented using the Eclipse Modeling Framework (EMF), and a graphical editor to manipulate

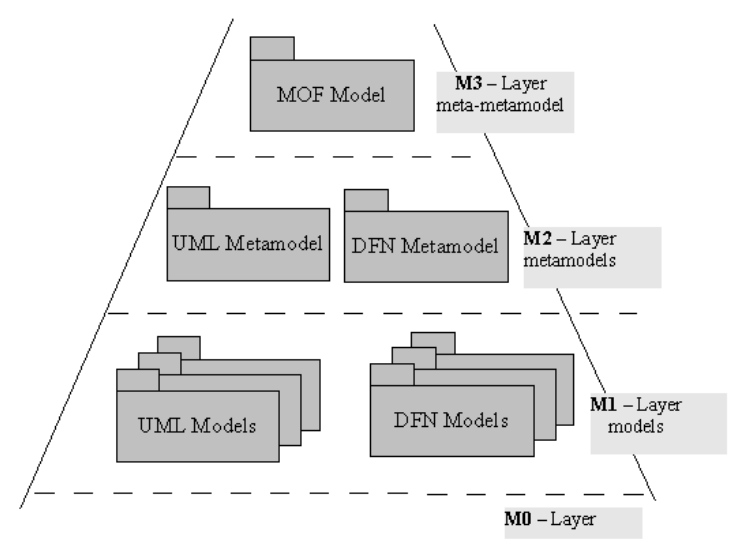

Figure 5: Relationship of MOF, UML and DFN 


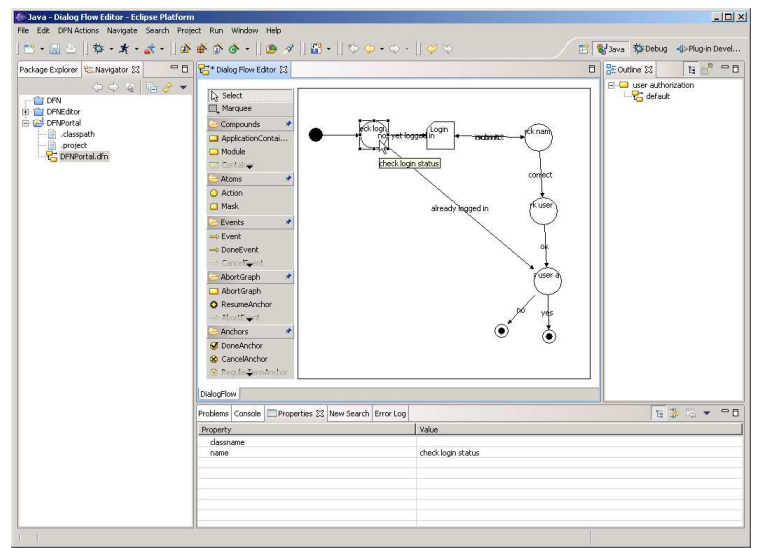

Figure 6: Model of the User Authorization module in the DFN CASE tool

DFN models, implemented using the Graphical Editing Framework (GEF). Since the EMF provides a powerful event mechanism, it was easy to integrate it into the MVC architecture of the GEF. This way, the editor can benefit from the metamodel with its default XMI serialization and validation.

One of the most important features of the DFN CASE tool is the validation of DFN models and highlighting any errors. This way, violations of the DFN rules can be recognized and eliminated without testing the application. MOF and EMF provide only few concepts to specify such constraints, which simplifies the development of metamodels, but limits the choices for validation. A commonly used concept are upper and lower bounds for attributes and associations in the metamodel, which can be validated automatically by the validation framework of EMF. The validation framework also provides basic mechanisms for the implementation of more complex constraints (e.g. the Object Constraint Language (OCL) in UML 2.0).

As an example, the screenshot of the DFN CASE tool in Figure 6 shows the graphical representation of the User Authorization module introduced in Figure 1. The following excerpt gives an impression of the serialized XMI representation of this dialog flow model:

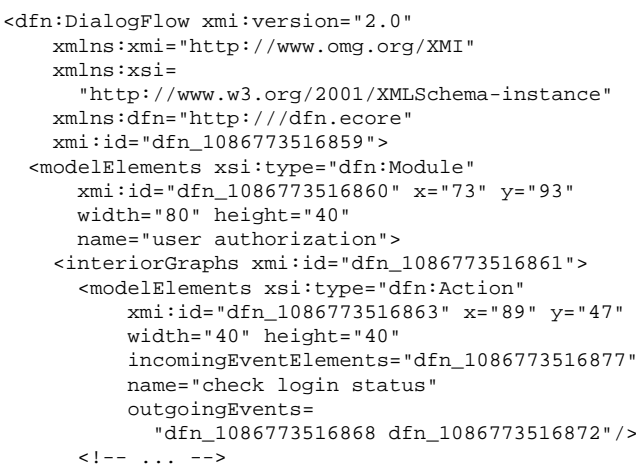

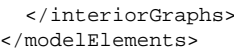

Given the DFN model in its serialized form, the XSLT transformation then produces DFSL documents as input for the DCF. As an example, the following is an excerpt from the User Authorization module's DFSL representation:

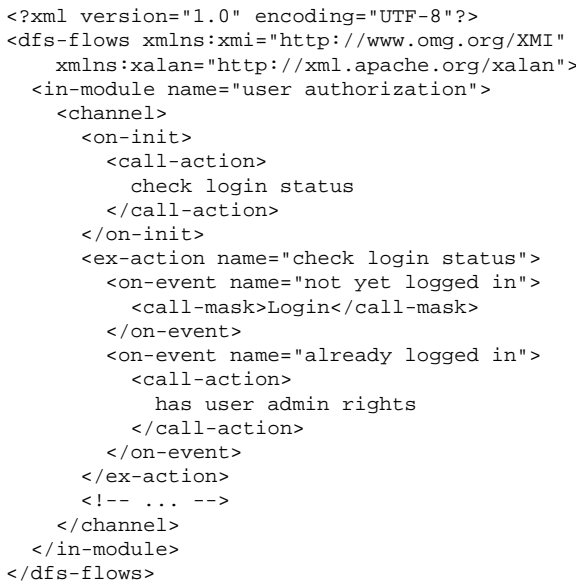

The requirements for the DFN CASE tool were derived from our Dialog-Driven Software Process Model (DDPM) that advocates modeling the dia$\log$ flows coarsely early in the software development process with a focus on modules and masks, and then refining it iteratively by adding actions that provide the application logic that is required to drive the envisioned user interface. Another source of requirements were our experiences from the development of AR$\mathrm{GuS}$, a web-based travel planning system that was still built without modeling tool support. The DFN CASE tool can be integrated smoothly into the DDPM without the need for additional processing steps.

\section{CONCLUSIONS}

In this paper, we presented issues and solutions that were encountered in the development of a metamodel for the Dialog Flow Notation (DFN). The aim of this metamodel is to increase the efficiency of the development process for web-based applications by providing tool support for the design and validation of dialog flow models and the automatic generation of dialog flow specification documents.

In order to achieve this goal, several issues had to be resolved: Firstly, we had to find a formal specification mechanism for the DFN that can be used for a complete description of DFN models with their rich syntax and semantics. This was accomplished by the choice of the MOF standard and its implementation in the EMF. Secondly, constraints imposed by the DFN rules had to be incorporated into the metamodel in 
order to enforce them in the process of model validation. These constraints were partly modelled as MOF constructs and partly implemented. Finally, a way for transforming valid DFN models into DFSL specifications had to be developed. This was accomplished by generating the XMI representation of the graphical model using the EMF and then translating it into DFSL using XSLT.

To formalize the DFN, we chose to develop a new metamodel instead of realizing it as a UML profile because of theoretical and practical reasons: The DFN has its well-defined domain and enough semantic distance from UML to justify an own metamodel, and UML 2.0 profiles are not supported properly by today's tools. We therefore implemented the DFN CASE tool as an Eclipse plugin to demonstrate the feasibility of the metamodelling approach for dialog flow specification. While the first practical experiences with the tool are promising, an experimental evaluation of its impact on the web application development process is still a topic of further research.

The development of the DFN CASE tool will continue in the future. It would be desirable to support more aspects of web application development, e.g. by generating further artefacts such as Java class stubs for implementing the actions and JavaServer Pages for implementing the masks. Although the full implementation of these artefacts will remain the developer's task, the CASE tool should be able to maintain the synchrony between model elements and their code representation, and allow prototypic dialog flow execution at design time. This way, the development process can be streamlined by avoiding frequent copying and pasting of class structures and lengthy testing of simple use cases.

Combining all possibilities offered by the DFN metamodel, the following scenario can be conceived: The development of a web application using the DFN is completely integrated into the Eclipse IDE. While the dialog flow is designed in the graphical editor, another DFN tool generates stubs for actions and masks in the same project. This yields a dialog flow that can be executed directly by a Dialog Control Framework plugin. While the dialog flow is refined and enhanced incrementally, the according artefacts are implemented in parallel. This would enable an agile development approach, which produces executable code very early, and is guided by the model of the dialog flow that is tailored to the users' requirements.

\section{ACKNOWLEDGMENTS}

The Chair of Applied Telematics/e-Business is endowed by Deutsche Telekom AG.

\section{REFERENCES}

Apache Project (2005). The Apache Struts web application framework. http://struts.apache.org.

Atkinson, C., Kühne, T., and Henderson-Sellers, B. (2000). To meta or not to meta. Journal of Object-Oriented Programming, 13(8):32-35.

Book, M. and Gruhn, V. (2003). A dialog control framework for hypertext-based applications. In Lin, H. and Ehrich, H., editors, Proceedings of the 3rd International Conference on Quality Software (QSIC 2003), pages 170-177. IEEE Computer Society Press.

Book, M. and Gruhn, V. (2004). Modeling web-based dia$\log$ flows for automatic dialog control. In 19th IEEE International Conference on Automated Software Engineering (ASE 2004), pages 100-109. IEEE Computer Society Press.

Ceri, S., Fraternali, P., and Bongio, A. (2000). Web Modeling Language (WebML): a modeling language for designing Web sites. Computer Networks, 33:137-157.

Conallen, J. (1999). Modeling web application architectures with UML. Communications of the ACM, 2(10):63-70.

Desfray, P. (2000). UML profiles versus metamodeling extensions... an ongoing debate. In UML In The Com Enterprise: Modeling CORBA, Components, XML/XMI And Metadata Workshop, Palm Springs.

Gorshkova, E. and Novikov, B. (2002). Exploiting UML extensibility in the design of web information systems. In Proceedings of the Fifth International Baltic Conference on Databases and Information Systems. Kluwer.

Harel, D. (1987). Statecharts: A visual formalism for complex systems. Science of Computer Programming, 8(3):231-274.

Krasner, G. (1988). A cookbook for using the model-viewcontroller user interface paradigm in Smalltalk. Journal of Object-Oriented Programming, 1(3):26-49.

Lüer, C. (2003). Evaluating the Eclipse platform as a composition environment. In 3rd International Workshop on Adoption-Centric Software Engineering (ACSE 2003), Portland.

Muller, P.-A., Studer, P., and Bezivin, J. (2003). Platform independent web application modelling. In Proceedings of the 6th International Conference on UML, Lecture Notes in Computer Science, volume 2863, pages 220 233. Springer.

Object Management Group (2002). Meta-object facility (MOF), v1.4. Technical report.

Schattkowsky, T. and Lohmann, M. (2002). Rapid development of modular dynamic web sites using UML. Proceedings of the 5th International Conference on UML, Lecture Notes in Computer Science, 2460:336-350.

Singh, I., Stearns, B., and Johnson, M. (2002). Designing Enterprise Applications with the J2EE Platform. Addison-Wesley, 2nd edition. 